\title{
Acompañamiento pedagógico y calidad de aprendizaje del área de comunicación en estudiantes del 6 to grado
}

\author{
Pedagogical Accompaniment and Learning Quality of the Communication Area in sixth grade \\ students
}

Flor Zoila Patricio Manrique ${ }^{1}$, Abelardo Rodolfo Campana Concha ${ }^{2}$

\begin{abstract}
RESUMEN
Objetivo: Analizar la relación presente en el acompañamiento pedagógico y el aprendizaje del Área de comunicación en estudiantes del 6to grado en las instituciones educativas nivel primaria, Cercado Huaral, 2018. Material y Método: Es un estudio correlacional, que consideró como instrumentos un cuestionario y acta de notas para el acompañamiento pedagógico un cuestionario y aprendizaje del área de comunicación, respectivamente, dirigido a a una muestra de 76 estudiantes, posteriormente procesada en el programa SPSS. Resultados: La mayoría de la muestra $(68,4 \%)$ califica como regular, en tanto que el $71,1 \%$ alcanzó el logro previsto en el aprendizaje del área de comunicación. Esto permite entender que el desarrollo del acompañamiento pedagógico tiene una aprobación media, lo cual está relacionado al aprendizaje de los estudiantes del curso de comunicación cuyas calificaciones no son destacadas. Conclusión: La satisfacción media del acompañamiento y sus dimensiones coordinación directivo docente, proceso enseñanza aprendizaje y asesoría personalizada, impide que los estudiantes tengan calificaciones destacadas en el curso de comunicación.
\end{abstract}

Palabras clave: Acompañamiento Pedagógico, Aprendizaje, Comunicación, Coordinación, Proceso, Asesoría.

\section{ABSTRACT}

Objective: To analyze the relationship present in the pedagogical accompaniment and the learning of the Communication Area in 6 th grade students in primary level educational institutions, Cercado Huaral, 2018. Material and Method: It is a correlational study, which considered as instruments a questionnaire and minutes for the pedagogical accompaniment a questionnaire and learning of the area of communication, respectively, addressed to a sample of 76 students, later processed in the SPSS program. Results: Most of the sample $(68,4 \%)$ qualifies as fair, while $71,1 \%$ reached the expected achievement in learning the area of communication. This allows us to understand that the development of the pedagogical accompaniment has a medium approval, which is related to the learning of the students of the communication course whose qualifications are not outstanding. Conclusion: The average satisfaction of the accompaniment and its dimensions, teacher management coordination, teaching-learning process and personalized advice, prevents students from having outstanding qualifications in the communication course.

Keywords: Pedagogical Accompaniment, Learning, Communication, Coordination, Process, Counseling.

\section{INTRODUCCIÓN}

Año tras año la demanda educativa se vuelve más exigente por parte de los estudiantes y de los mismos docentes, si bien estos últimos intentan capacitar, estos conocimientos serán comprobados en la práctica, una manera de hacer un seguimiento de su rendimiento en las aulas es el acompañamiento pedagógico.

Previos a enfocarse en el acompañamiento pedagógico, el Ministerio de Educación aplicaba el Programa Estratégico Logros de Aprendizaje al finalizar el III Ciclo de Educación Básica Regular (PELA), creado en el 2008, el cual tenía por finalidad: "revertir los resultados de las evaluaciones nacionales que mostraron problemas importantes de calidad en los logros de aprendizajes de los estudiantes, tanto en comprensión de textos como en habilidades matemática" (Minedu, 2011).

En otras palabras, para trabajar propiamente con el acompañamiento pedagógico, tuvo que pasar por ciertas etapas, para tratar de adaptar el programa a las necesidades de los estudiantes.
Según Rodríguez, Leyva y Hopkins (2016) el acompañamiento pedagógico:

"Era parte esencial del PELA. Desde su creación, al PELA se le han ido agregando otros componentes que son complementarios entre sí y el propio acompañamiento pedagógico se ha visto modificado. En ese sentido se podría decir que el acompañamiento pedagógico tiene tres períodos: (i) desde su creación y hasta el cambio de gobierno en 2011, (ii) el periodo de revisión y rediseño entre 2011 y 2012, y (iii) el de la implementación del rediseño a partir del 2013" (p.4).

Según la Resolución Viceministerial № 169 -2019Minedu que establece la "Norma que establece disposiciones para el Desarrollo del Acompañamiento Pedagógico en la Educación Básica para el año 2019", el acompañante pedagógico se caracteriza por ser:

"Un profesional que cuenta con título pedagógico, con certificación en rúbricas y con el perfil requerido para desarrollar las estrategias del programa de formación docente con acompañamiento pedagógico: visitas en 
aula, grupos de inter aprendizajes y talleres. Asume al docente como un profesional de la educación que reflexiona sobre sus fortalezas y dificultades, que tiene capacidad de aprender de su propia práctica y mejorar progresivamente sus competencias, según el nivel en el que se encuentre" (p.4)

Sin embargo, algunos docentes de la población docente seleccionado notaron que en este proceso no se están enfocando en sus fortalezas, lo que genera incomodidad e insatisfacción.

En el área de comunicación, las instituciones educativas se basan en el Currículo Nacional que considera tres competencias: se comunica oralmente, lee diversos textos y escribe diversos textos. Sin embargo los estudiantes aun no alcanzan los promedios académicos deseados.

Minedu (2018) indica que en la evaluación PISA de ese año, los resultados en lectura se alcanzaron los 401, tres puntos en comparación a los resultados del 2015, sin embargo, esto solo representa el Nivel 1a, siendo el máximo el nivel 6 . Ante ello, planteamos la necesidad de seguir perfeccionando la actividad docente, realizando los seguimientos respectivos, en beneficio de los estudiantes de educación básica.

El plantear esta investigación fue resultado de lo encontrados en Ruiz (2015) quien destaca la importancia del acompañamiento pedagógico para el mejoramiento de su desempeño, sin embargo lo que encontró fue que muchas veces se realizan sin un determinado plan y objetivo, descuidando las necesidades docentes de las distintas áreas académicas, asimismo considera necesario que se respete cada etapa del acompañamiento.

En cuanto al aprendizaje del curso de comunicación, encontramos a Rivera (2012) quien encontró que es necesario un clima idóneo dentro del aula ya que "las buenas relaciones humanas, el orden y respeto mutuo, el ejercicio pedagógico del tratamiento de la indisciplina y los valores" ayudan al facilitamiento del aprendizaje, sin embargo considera que para conseguir ello es necesario la exigencia del centro educativo para su cumplimiento.

El objetivo de la investigación es analizar la relación entre el acompañamiento pedagógico y aprendizaje del área de comunicación.

\section{MÉTODOS}

Corresponde a la investigación del enfoque cuantitativo, nivel explicativo. Se realizó un análisis correlacional entre las variables presentadas. La población estuvo representada por 210 estudiantes, se seleccionó 76 como muestra, los instrumentos para su análisis, fueron cuestionario y acta de notas. Procesado estadísticamente mediante SPSS versión 21.

\section{RESULTADOS}

Tabla 1

Frecuencias Acompañamiento Pedagógico

\begin{tabular}{llccc}
\hline $\begin{array}{l}\text { Acompañamiento } \\
\text { Pedagógico }\end{array}$ & Frecuencia & $\%$ & $\begin{array}{c}\% \\
\text { acumulado }\end{array}$ \\
\hline \multirow{3}{*}{ Válidos } & $\begin{array}{l}\text { Poco } \\
\text { adecuado }\end{array}$ & 24 & 31,6 & 31,6 \\
\cline { 2 - 5 } & Regular & 52 & 68,4 & 100,0 \\
\cline { 2 - 5 } & Total & 76 & 100,0 & \\
\hline
\end{tabular}

El 68,4\% considera que el acompañamiento pedagógico aplicado en la institución es regular, mientras que el $31,6 \%$ lo considera poco adecuado, con lo cual evidenciamos que existen aspectos de inconformidad por parte de la muestra.

\section{Tabla 2}

Frecuencias aprendizaje del área de comunicación

\begin{tabular}{llllr}
\hline $\begin{array}{l}\text { Área de } \\
\text { Comunicación }\end{array}$ & Frecuencia & $\%$ & $\%$ acumulado \\
\hline \multirow{3}{*}{ Válidos } & $\begin{array}{l}\text { En } \\
\text { proceso }\end{array}$ & 22 & 28,9 & 28,9 \\
\cline { 2 - 5 } & $\begin{array}{l}\text { Logro } \\
\text { previsto }\end{array}$ & 54 & 71,1 & 100,0 \\
\cline { 2 - 5 } & Total & 76 & 100,0 & \\
\hline
\end{tabular}

Se comprueba que el $71,1 \%$ de los estudiantes de sexto grado alcanzan el logro previsto en comunicación, mientras $28,9 \%$ está en proceso, con lo cual evidenciamos la ausencia de calificaciones destacadas.

\section{Prueba hipótesis general}

HA. El acompañamiento pedagógico se relaciona con el aprendizaje del Área de comunicación en estudiantes de primaria.

HO. El acompañamiento pedagógico no se relaciona con el aprendizaje del Área de comunicación en estudiantes de primaria.

\section{Tabla 3}

Correlación hipótesis general

\begin{tabular}{lllr}
\hline Correlación & \multicolumn{2}{c}{$\begin{array}{c}\text { Acompañamiento } \\
\text { pedagógico }\end{array}$} \\
\hline & $\begin{array}{l}\text { Coeficiente de } \\
\text { correlación }\end{array}$ & 0,752 \\
$\begin{array}{l}\text { Rho de } \\
\text { Spearman }\end{array}$ & $\begin{array}{l}\text { Aprendizaje } \\
\text { del área de } \\
\text { comunicación }\end{array}$ & Sig. (bilateral) & 0,000 \\
& $\mathrm{~N}$ & 76
\end{tabular}

Con margen de error al $5 \%$, se halló una correlación buena de Rho $=0,752$ y el $p=0,000$, que confirma la aceptación de la $\mathrm{Ha}$. Los resultados mayoritarios dan cuenta de que la regular aplicación de la variable 1 está relacionada al logro previsto del curso de comunicación. 


\section{Prueba hipótesis específica 1}

H1. La coordinación directivo docente se relaciona con el aprendizaje del Área de comunicación en estudiantes del 6 to grado.

Ho. La coordinación directivo docente no se relaciona con el aprendizaje del Área de comunicación en estudiantes del 6 to grado.

\section{Tabla 4}

Correlación hipótesis específica 1

\begin{tabular}{lllr}
\hline \multicolumn{1}{c}{ Correlación } & \multicolumn{2}{c}{$\begin{array}{c}\text { Coordinación } \\
\text { directivo docente }\end{array}$} \\
\hline & $\begin{array}{l}\text { Coeficiente de } \\
\text { correlación }\end{array}$ & 0,614 \\
$\begin{array}{l}\text { Rho de } \\
\text { Spearman }\end{array}$ & $\begin{array}{l}\text { Aprendizaje } \\
\text { del área de } \\
\text { comunicación }\end{array}$ & Sig. (bilateral) & 0,000 \\
& $\mathrm{~N}$ & 76 \\
\hline
\end{tabular}

Aplicando el Rho de Spearman se encontró una correlación buena de Rho $=0,614$ y el $p=0,000$ con lo cual se da aceptación de la hipótesis alterna, cuyos resultados mayoritarios dan cuenta que a regular aplicación de la coordinación directivo docente está involucrado al logro previsto en el aprendizaje de este curso.

\section{Prueba hipótesis específica 2}

H2. El proceso enseñanza aprendizaje se relaciona con el aprendizaje del Área de comunicación en estudiantes del 6to grado.

Ho. El proceso enseñanza aprendizaje no se relaciona con el aprendizaje del Área de comunicación en estudiantes del 6to grado.

\section{Tabla 5}

Correlación hipótesis específica 2

\begin{tabular}{lllr}
\hline \multicolumn{1}{c}{ Correlación } & \multicolumn{2}{c}{$\begin{array}{c}\text { Proceso enseñanza } \\
\text { aprendizaje }\end{array}$} \\
\hline & \multicolumn{2}{c}{$\begin{array}{l}\text { Coeficiente de } \\
\text { correlación }\end{array}$} & 0,563 \\
$\begin{array}{l}\text { Rho de } \\
\text { Spearman }\end{array}$ & $\begin{array}{l}\text { Aprendizaje } \\
\text { del área de } \\
\text { comunicación }\end{array}$ & Sig. (bilateral) & 0,000 \\
& $\mathrm{~N}$ & 76
\end{tabular}

Se identificó una correlación moderada de Rho = 0,563 y el $p=0,000$ datos que comprueban la aceptación de la hipótesis alterna, cuyos resultados mayoritarios dan cuenta de que la regular aplicación del proceso enseñanza aprendizaje está vinculado al logro previsto en el aprendizaje de este curso.

\section{Prueba hipótesis específica 3}

H3. La asesoría personalizada se relaciona con el aprendizaje del Área de comunicación en estudiantes de primaria.

Ho. La asesoría personalizada no se relaciona con el aprendizaje del Área de comunicación en estudiantes de primaria.

\section{Tabla 6}

Correlación hipótesis específica 3

\begin{tabular}{lllr}
\hline & \multicolumn{3}{c}{$\begin{array}{c}\text { Asesoría } \\
\text { personalizada }\end{array}$} \\
\hline & $\begin{array}{l}\text { Coeficiente de } \\
\text { correlación }\end{array}$ & 0,628 \\
$\begin{array}{l}\text { Rho de } \\
\text { Spearman }\end{array}$ & $\begin{array}{l}\text { Aprendizaje } \\
\text { del área de } \\
\text { comunicación }\end{array}$ & Sig. (bilateral) & 0,000 \\
& $\mathrm{~N}$ & 76 \\
\hline
\end{tabular}

Se identificó una correlación buena de Rho $=0,628$ y el $p$ $=0,000$ lo cual nos permite rechazar la hipótesis nula y aceptar la hipótesis alterna.

\section{DISCUSIÓN}

En la hipótesis general se encontró una correlación buena de Rho $=0,752$ entre las variables estudiadas. En la variable acompañamiento pedagógico la mayoría de la muestra representado por el 68,4\% lo califica de regular y el 31,6\% poco adecuado. Variable aprendizaje área de comunicación, el análisis de las notas de los estudiantes evidenció $71,1 \%$ se ubican en logro previsto y $28,9 \%$ el nivel en proceso. Esto permite entender que el desarrollo del acompañamiento pedagógico tiene una aprobación media, sin embargo un porcentaje lo desaprueba lo cual está relacionado al aprendizaje de los estudiantes del curso de comunicación cuyas calificaciones no son destacadas. Haciendo un comparativo en los resultados encontramos los de Portela (2014) quien comprobó que un seguimiento exhaustivo y cumpliendo los parámetros establecidos del acompañamiento pedagógico, todo el personal docente cumplirá con su objetivo que desempeñarse idóneamente, en un principio solo lo conseguía un porcentaje, sin embargo el seguimiento y planteamiento de mejora logró ello, en cambio nosotros evidenciamos una satisfacción regular ya que no existe la comunicación adecuada y las reuniones de asesoría resultan insuficiente, dando a entender que si se trabaja con mayor conciencia o implementando programas esta situación se puede revertir.

En cuanto al aprendizaje del aprendizaje del área de comunicación, Gamarra (2015) destaca que son muchas competencias que se debe desarrollar en las distintas unidades, sin embargo en las actividades asignadas resultan insuficientes, generando, según su opinión, un desbalance entre actividades y capacidades, lo cual resulta cierto si es que los docentes no innovan en metodologías, tareas y otros elementos los estudiantes no aprenderán como se debe lo cual solo les permitirá 
alcanzar promedios de logro previsto o en proceso.

La hipótesis específica uno, identificó una correlación buena de Rho $=0,614$ que avalan la relación de la dimensión coordinación directivo - docente con la segunda variable. En cuanto a la mencionada dimensión, el $68,4 \%$ considera regular, $25,0 \%$ poco adecuado; la variable aprendizaje del área comunicación se evidenció $71,1 \%$ se encuentra en logro previsto y $28,9 \%$ en proceso. La percepción que se tiene, es que la comunicación entre los directores y docentes sobre las actividades durante las visitas son inefectivas, lo cual de alguna forma perjudica a los estudiantes en sus calificaciones. Realizando contraste con Gamarra (2015) considera existe desbalance en las actividades institucionales, repercutiendo el aprendizaje en comunicación, situación comprobada entre coordinación directivo y docente calificado de satisfacción media, no permite el desarrollo del aprendizaje.

La hipótesis específica dos, halló una correlación moderada de $\mathrm{Rho}=0,563$ que explica la relación entre la dimensión proceso enseñanza - aprendizaje con la segunda variable. Esta dimensión, 57,9\% consideran regular, 36,8\% poco adecuado; para aprendizaje comunicación, $71,1 \%$ están en logro previsto y $28,9 \%$ en proceso. Manifestándose que los docentes no sientes que el proceso de observación, organización y análisis no son los adecuados para mejorar y ayudar en sus actividades académicas, lo cual se relaciona en los resultados finales en el curso de comunicación. Por su parte, Rengifo (2016) considera necesario la creación de un programa estratégico de logros de aprendizaje para el área de comunicación, aunque los resultados no utilicen las mismas variables, se entiende la necesidad de buscar elementos que fortalezcan el aprendizaje del curso.

La hipótesis específica tres, encontró una correlación buena de Rho $=0,628$ que manifiesta la relación entre la tercera dimensión del acompañamiento pedagógico con la segunda variable. La asesoría personalizada es referida por el $61,8 \%$ de regular y el $34,2 \%$ de poco adecuado, mientras aprendizaje en comunicación en donde el análisis de las notas de los estudiantes evidenció que el $71,1 \%$ muestran logro previsto y el $28,9 \%$ el nivel en proceso. Con lo cual se comprende que las reuniones luego de haberse realizado el acompañamiento no tienen intermedia, aunque evidencia un grado de inconformidad lo cual está relacionado a las calificaciones del curso en mención.

En las investigaciones de Mairena (2015) se explicó la insatisfacción de la muestra con respecto a la forma con la que se desarrolla el acompañamiento de la institución, entre estas las asesorías que dificultan la retroalimentación para el mejoramiento académico, aunque en esta parte nuestra muestra considera regular, la otra parte encuentra dificultades de su desarrollo, confirmando nuevamente las carencias y la importancia para garantizar el aprendizaje.

\section{CONCLUSIONES}

Se halló satisfacción media respecto al acompañamiento y desaprobación por algunos docentes impide los estudiantes tengan calificaciones destacadas en el curso de comunicación.

El proceso enseñanza aprendizaje no fue óptimo, para que aprendan el área de comunicación adecuadamente.

Por otra parte, la forma de realizar acciones de observación del participante, de igual modo refleja bajos aprendizajes en comunicación.

La asesoría personalizada de igual modo no fue lo esperando, incumpliendo la labor docente que implica en apoyar en forma individual sobre todo a los estudiantes que tienen dificultades de aprendizaje.

\section{REFERENCIAS BIBLIOGRÁFICAS}

Gamarra, A. (2015). Las actividades de aprendizaje en el desarrollo de las competencias del área de comunicación en las unidades de aprendizaje del nivel secundario. PUCP.

Mairena, E. (2015). Acompañamiento pedagógico y desempeño de los docentes noveles en los departamentos de física y tecnología educativa de la facultad de educación e idiomas. http://repositorio.unan.edu.ni/1434/1/4428.pdf.

Minedu (2018). Evaluación PISA 2018. http://umc.minedu.gob.pe/resultadospisa2018/.

Portela, A. (2014). Implementación de un instrumento de evaluación de desempeño laboral docente y su impacto en el clima organizacional del colegio Colombo Galés (Guayamaral - Bogotá) Plan de $m$ e $j$ o $\quad r \quad$ a $m$ i $\quad$ e $n \quad n \quad t \quad o$. https://repository.unilibre.edu.co/bitstream/handle/1090 1/8650/PROYECTO\%20DE\%20GRADO.pdf?sequence $=1$ \&isAllowed $=\mathrm{y}$.

Rengifo, A. (2016). Evaluación del programa Estratégico logros de aprendizaje y su relación con los aprendizajes significativos en las áreas de comunicación y matemática de los estudiantes del segundo grado de primaria en la l.E 0180 Señor de los Milagros.

Resolución Viceministerial N 169 -2019- Minedu. Diario Oficial EI Peruano, Lima, Perú, 13 de julio de 2019.

Rivera, M. (2012). Clima de aula y logros de aprendizaje en Comunicación Integral del Quinto Grado de 4 Instituciones Educativas de Ventanilla.

Rodríguez, J., Leyva, J. y Hopkins, A. (2016). El efecto del programa acompañamiento pedagógico sobre los rendimientos de los estudiantes de escuelas públicas rurales del Perú. Editorial PUCP.

Ruíz, D. (2015). Incidencia del Acompañamiento 
Pedagógico en el desempeño de los docentes de educación secundaria del Colegio Liceo Franciscano, ubicado en el distrito No. 1 de la ciudad de Managua. http://repositorio.unan.edu.ni/1266/1/47422.pdf. 